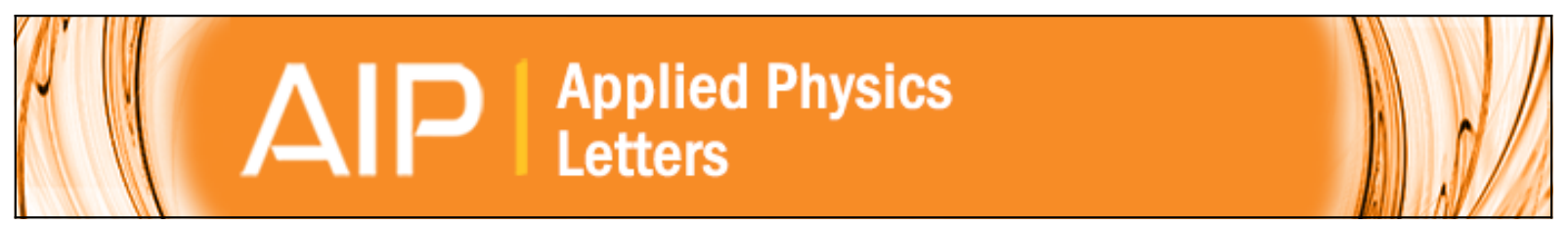

\title{
Photoluminescence studies of type-II CdSe/CdTe superlattices
}

Jing-Jing Li, Leijun Yin, Shane R. Johnson, B. J. Skromme, Shumin Wang, Xinyu Liu, Ding Ding, Cun-Zheng Ning, Jacek K. Furdyna, and Yong-Hang Zhang

Citation: Applied Physics Letters 101, 061915 (2012); doi: 10.1063/1.4745199

View online: http://dx.doi.org/10.1063/1.4745199

View Table of Contents: http://scitation.aip.org/content/aip/journal/apl/101/6?ver=pdfcov

Published by the AIP Publishing

\section{Articles you may be interested in}

Time-resolved and excitation-dependent photoluminescence study of $\mathrm{CdTe} / \mathrm{MgCdTe}$ double heterostructures grown by molecular beam epitaxy

J. Vac. Sci. Technol. B 32, 040601 (2014); 10.1116/1.4878317

Optimization of growth conditions of type-II $\mathrm{Zn}(\mathrm{Cd}) \mathrm{Te} / \mathrm{ZnCdSe}$ submonolayer quantum dot superlattices for intermediate band solar cells

J. Vac. Sci. Technol. B 31, 03C119 (2013); 10.1116/1.4797486

Radiative transitions in stacked type-II ZnMgTe quantum dots embedded in ZnSe

J. Appl. Phys. 112, 063521 (2012); 10.1063/1.4754451

CdSe/CdTe type-II superlattices grown on GaSb (001) substrates by molecular beam epitaxy

Appl. Phys. Lett. 100, 121908 (2012); 10.1063/1.3697676

Coexistence of type-l and type-II band lineups in Cd ( Te, Se )/Zn Se quantum-dot structures

Appl. Phys. Lett. 89, 123110 (2006); 10.1063/1.2355439

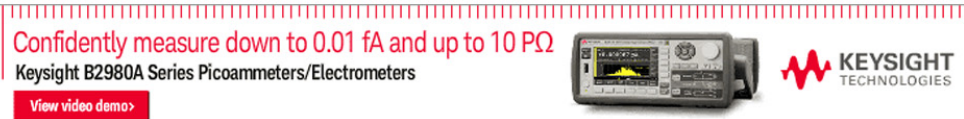




\title{
Photoluminescence studies of type-II CdSe/CdTe superlattices
}

\author{
Jing-Jing Li, ${ }^{1,2}$ Leijun Yin, ${ }^{1,3}$ Shane R. Johnson, ${ }^{1,2}$ B. J. Skromme, ${ }^{2}$ Shumin Wang, ${ }^{1,2}$ \\ Xinyu Liu, ${ }^{4}$ Ding Ding, ${ }^{1,2}$ Cun-Zheng Ning, ${ }^{1,2}$ Jacek K. Furdyna, ${ }^{4}$ \\ and Yong-Hang Zhang ${ }^{1,2, a)}$ \\ ${ }^{1}$ Center for Photonics Innovation, Arizona State University, Tempe, Arizona 85287-5706, USA \\ ${ }^{2}$ School of Electrical, Computer, and Energy Engineering, Arizona State University, Tempe, \\ Arizona 85287-5706, USA \\ ${ }^{3}$ Department of Physics, Arizona State University, Tempe, Arizona 85287-1504, USA \\ ${ }^{4}$ Department of Physics, University of Notre Dame, Notre Dame, Indiana 46556, USA
}

(Received 14 May 2012; accepted 27 July 2012; published online 9 August 2012)

\begin{abstract}
$\mathrm{CdSe} / \mathrm{CdTe}$ type-II superlattices grown on GaSb substrates by molecular beam epitaxy are studied using time-resolved and steady-state photoluminescence (PL) spectroscopy at $10 \mathrm{~K}$. The relatively long carrier lifetime of $188 \mathrm{~ns}$ observed in time-resolved PL measurements shows good material quality. The steady-state PL peak position exhibits a blue shift with increasing excess carrier concentration. Self-consistent solutions of the Schrödinger and Poisson equations show that this effect can be explained by band bending as a result of the spatial separation of electrons and holes, which is critical confirmation of a strong type-II band edge alignment between CdSe and CdTe. (C) 2012 American Institute of Physics. [http://dx.doi.org/10.1063/1.4745199]
\end{abstract}

The monolithic integration of $6.1 \AA$ II-VI $(\mathrm{MgZnCd})$ (SeTe) and III-V (AlGaIn)(AsSb) semiconductors on commercially available GaSb or InAs substrates offers the freedom to integrate many photonic and electronic devices onto a single substrate. ${ }^{1-3}$ Because these materials can be grown lattice matched on $\mathrm{GaSb}$ or InAs substrates, high quality materials can be obtained with minimal misfit dislocations to ensure the best possible device performance. In particular, $\mathrm{CdSe} / \mathrm{CdTe}$ superlattices have recently been grown on $\mathrm{GaSb}$ substrates using molecular beam epitaxy. ${ }^{4}$ The excellent structural and optical properties of these superlattices make them suitable for applications such as multi-junction solar cells, light emitting diodes, and photodetectors. The $\mathrm{CdSe} /$ CdTe heterostructure has a type-II band edge alignment, and therefore offers some unique optical properties. ${ }^{4}$ This paper reports a detailed study of the optical properties of the typeII superlattices using time-resolved and steady-state photoluminescence (PL) spectroscopy. The superlattice sample used as an example consists of 50 periods of alternating CdSe and $\mathrm{CdTe}$ layers with thicknesses of $6.0 \pm 0.1 \mathrm{~nm}$ and $0.8 \pm 0.1 \mathrm{~nm}$, respectively, on a ZnTe buffer layer grown using molecular beam epitaxy on the GaSb substrate. Detailed growth conditions and structural characterization results are reported in Ref. 4.

The time-dependent PL decay of the superlattice was measured at $10 \mathrm{~K}$ using time-correlated single photon counting and is shown in Fig. 1 for two optical excitation intensities. The excitation source was a $405 \mathrm{~nm}$ pulsed laser with a 50 ps pulse width and a $2.5 \mathrm{MHz}$ repetition rate, and the detector was a Hamamatsu H10330-75 near infrared photomultiplier tube. Assuming there is no carrier recombination during the laser pulse, the initial excess carrier concentrations are estimated to be $5 \times 10^{18} \pm 1 \times 10^{18} \mathrm{~cm}^{-3}$ (red curve) and $4 \times 10^{17} \pm 1 \times 10^{17} \mathrm{~cm}^{-3}$ (blue curve) using the

\footnotetext{
${ }^{\text {a) }}$ Author to whom correspondence should be addressed. Electronic mail: yhzhang@asu.edu.
}

measured laser peak powers of $554 \pm 20 \mathrm{~mW}$ and $44 \pm 2 \mathrm{~mW}$, laser spot diameter of $15 \pm 2 \mu \mathrm{m}$, and the absorption coefficient of $1.5 \times 10^{5} \mathrm{~cm}^{-1}$ measured using UV-visible variable-angle spectroscopic ellipsometry. The laser spot size is estimated by imaging the focused laser spot on a reference sample using an Indigo Alpha NIR camera on a micro-PL setup, with an accuracy of about $1 \mu \mathrm{m}$ as limited by the camera pixel size.

The PL decay evolves from a steep initial descent with a rapidly increasing instantaneous lifetime in the first $75 \mathrm{~ns}$ to a gradual decay with a single exponential tail. The strong dependence of carrier lifetime on the excess carrier concentration in the initial decay can be partly attributed to concentration-dependent recombination mechanisms such as radiative and Auger recombination. ${ }^{5}$ However, the similarity in the initial slopes of the PL decay curves, in spite of the very different initial excess carrier concentrations, suggests that other mechanisms are also involved in the initial steep PL decay, e.g., carrier diffusion and the direct band-to-band

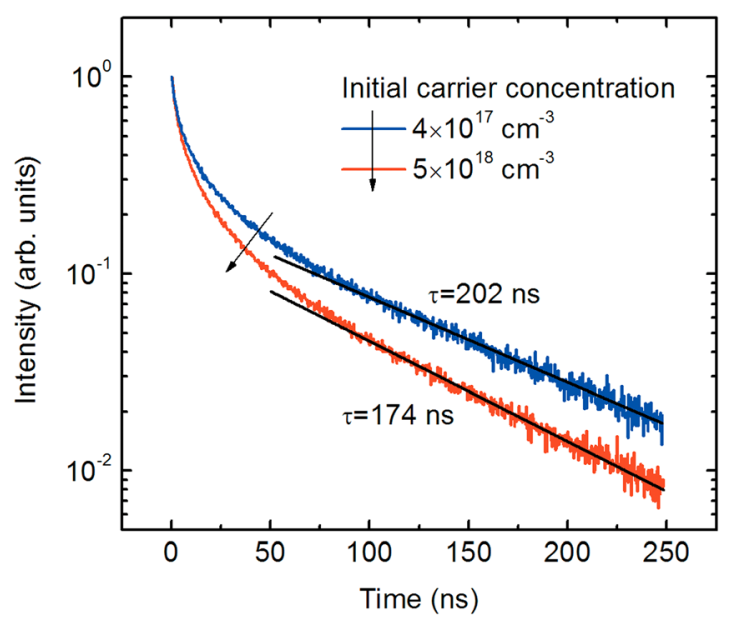

FIG. 1. Time-resolved photoluminescence decays measured at $10 \mathrm{~K}$ with initial carrier concentrations of $5 \times 10^{18} \mathrm{~cm}^{-3}$ and $4 \times 10^{17} \mathrm{~cm}^{-3}$, respectively. 
transition in CdSe. The latter was directly observed in steady-state PL on a similar sample.

The excess carrier concentrations at the onset of the exponential decay tails are on the order of $10^{18} \mathrm{~cm}^{-3}$ (red curve) and $10^{17} \mathrm{~cm}^{-3}$ (blue curve), respectively. The net background doping concentration at room temperature is estimated to be below $10^{17} \mathrm{~cm}^{-3}$ from the free carrier absorption measured using an infrared variable angle spectroscopic ellipsometer. Therefore, the excess carrier concentrations at the onset of the exponential decay tails are assumed to be much larger than the background carrier concentration considering carrier freeze-out at $10 \mathrm{~K}$. In this case, the constant lifetimes in the exponential decay tails can be attributed to Shockley-Read-Hall (SRH) recombination, and the PL intensity has a quadratic dependence on the excess carrier concentration. The PL decay lifetimes in the exponential decay tails are fit as $174 \pm 6 \mathrm{~ns}$ and $202 \pm 7 \mathrm{~ns}$ for the initial excess carrier concentrations of $5 \times 10^{18} \mathrm{~cm}^{-3}$ and $4 \times 10^{17} \mathrm{~cm}^{-3}$, respectively. The slight difference between the observed lifetimes may be due to the limited time range taken in the measurements, and the SRH lifetime is taken as $188 \pm 5 \mathrm{~ns}$ from the average of those fit values.

The steady-state PL of the superlattice was measured at $10 \mathrm{~K}$ with excess carrier concentrations ranging from approximately $9 \times 10^{15} \mathrm{~cm}^{-3}$ to $6 \times 10^{18} \mathrm{~cm}^{-3}$. The sample was excited by a $488 \mathrm{~nm}$ Ar-ion laser with a focused spot diameter of $134 \pm 2 \mu \mathrm{m}$ measured using the knife-edge method, and the PL signals were collected using a grating monochromator and measured using a $\mathrm{Ge}$ detector cooled to liquid nitrogen temperature. As shown in Fig. 2(a), the PL peak energy of the superlattice is smaller than the bandgap energy of either CdTe $(1.61 \mathrm{eV})^{9,12,13}$ or cubic $\mathrm{CdSe}(1.76 \mathrm{eV}),{ }^{9,10}$ suggesting a typeII band edge alignment. Furthermore, the PL peak shifts to higher energy as the excess carrier concentration increases. This blue shift is proposed to be caused by the spatial separation of electrons and holes generated by the optical excitation in the type-II superlattice. Figure 2(b) shows the calculated band edge alignments, the superlattice minibands, and the corresponding ground state probability densities of the conduc- tion band and heavy-hole band at an excess carrier concentration of $6 \times 10^{18} \mathrm{~cm}^{-3}$. (Details of the calculations are described below.) The electrons and holes are spatially separated as shown by their probability densities, and their Coulomb attraction pulls them toward the interfaces of the two materials. Consequently, the bands are bent down towards the interfaces, pushing the minibands to higher energies as the excess carrier concentration increases with increasing excitation power density. A blue shift in the PL peak should, therefore, result from the enhanced band bending caused by the increased excess carrier concentration.

Pronounced band bending of this type has been observed primarily in type-II quantum wells and quantum dots. ${ }^{6,7} \mathrm{In}$ superlattices, however, the coupling between the quantum wells tends to reduce the charge localization and separation as shown by the probability densities in Fig. 2(b). To confirm the band bending effect in the type-II superlattices, selfconsistent solutions of the Schrödinger and Poisson equations $^{8}$ are performed to model the increase of the ground state transition energy with the accumulation of excess carriers.

The modeling starts with the calculation of strain effects on the band edges. In $\mathrm{CdSe} / \mathrm{CdTe}$ superlattices, the $\mathrm{CdSe}$ layer is tensilely strained, while the CdTe layer is compressively strained to match the GaSb lattice constant. The strain effects on the band edges are caused by a superposition of the hydrostatic strain and shear strain. The hydrostatic strain shifts the conduction band and valence band edges, while the shear strain splits the heavy-hole and light-hole bands. The superlattice minibands are formed from a hybridization of bound states of the quantum wells coupled through the barriers, which can be obtained by solving the Schrödinger-like envelope function equation

$$
-\frac{\hbar^{2}}{2} \frac{d}{d z}\left[\frac{1}{m(z)} \frac{d}{d z}\right] \Psi(z)+\left[V_{\mathrm{SL}}(z)-V_{\mathrm{E}}(z)\right] \Psi(z)=E \Psi(z),
$$

where $m(z)$ is the effective mass in the growth direction $z$, $\Psi(z)$ is the wave function of the energy state $E$, and $V_{\mathrm{SL}}(z)$
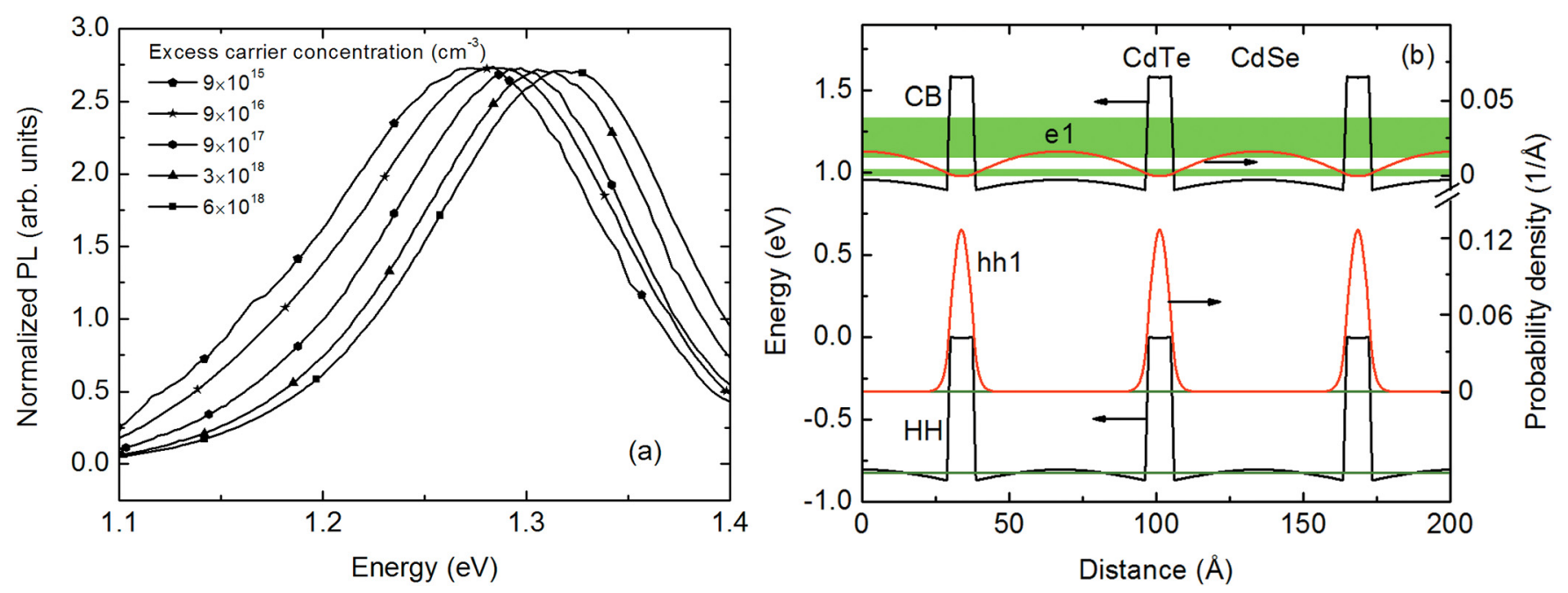

FIG. 2. (a) Steady-state photoluminescence spectra measured at $10 \mathrm{~K}$ with estimated excess carrier concentrations ranging from $9 \times 10^{15} \mathrm{~cm}^{-3}$ to $6 \times 10^{18} \mathrm{~cm}^{-3}$. (b) Calculated band edge alignments (black), minibands (green), and the corresponding ground state probability densities of the conduction band and heavy-hole band (red) at a carrier concentration of $6 \times 10^{18} \mathrm{~cm}^{-3}$. 
and $V_{\mathrm{E}}(z)$ are, respectively, the band edge alignment of the superlattice and the electrostatic potential induced by the charges. For a superlattice of $N$ periods of a period $L$, the wave vector $q$ has $N$ values in the first Brillouin zone with a spacing of $2 \pi / N L$ according to the envelope function approximation and the cyclic boundary condition. The energy states corresponding to $+q$ and $-q$ are degenerate. Equation (1) is solved, respectively, for the conduction band, the heavy-hole band, and the light-hole band using the propagation matrix method, in order to obtain the energy states and wave functions corresponding to the wave vectors.

The charges accumulated under optical excitation generate an electrostatic potential $V_{\mathrm{E}}(z)$ as described by the Poisson equation (neglecting ionized dopants)

$$
\frac{d}{d z}\left[\varepsilon(z) \frac{d}{d z}\right] V_{\mathrm{E}}(z)=-|e|[p(z)-n(z)],
$$

where $\varepsilon(z)$ is the dielectric function and $n(z)$ and $p(z)$ are the electron and hole concentrations. The electron concentration $n(z)$ is

$$
n(z)=\sum_{m} \sum_{n}\left|\Psi_{n m}(z)\right|^{2} N_{n m},
$$

where $\left|\Psi_{n m}(z)\right|^{2}$ and $N_{n m}$ are the electron probability density and the sheet concentration in the $n$th energy state $E_{n m}$ of the $m$ th miniband in the conduction band. The electron sheet concentration for the energy level $E_{n m}$ is

$$
N_{n m}=\frac{k_{\mathrm{B}} T m_{e}}{\pi \hbar^{2}} \ln \left(1+e^{\left[F_{\mathrm{N}}-E_{n m}\right] / k_{\mathrm{B}} T}\right) .
$$

The electron quasi-Fermi level $F_{\mathrm{N}}$ can thus be found using Eqs. (3) and (4) assuming the incident light is evenly absorbed in every period of the superlattices. The hole quasiFermi level $F_{\mathrm{P}}$ is obtained similarly considering both the heavy-hole and light-hole bands. Consequently, the electron, heavy-hole, and light-hole concentrations are obtained, and Eq. (2) is solved for the electrostatic potential $V_{\mathrm{E}}(z)$. This procedure of solving the coupled Schrödinger and Poisson equations is iterated until it converges.

The material parameters used in the calculations are shown in Table I. The absorption coefficient and the surface reflectivity at the $488 \mathrm{~nm}$ laser wavelength are $10^{5} \mathrm{~cm}^{-1}$ and $28 \%$, respectively, as estimated from the ellipsometry measurements. The electron and hole concentrations are assumed for simplicity to be the same in each period of the superlat- tice that contributes to the PL. The surface recombination is not expected to be significant because of the strong hole confinement in the structure. The carrier lifetime used in the calculations is $188 \mathrm{~ns}$ as obtained from the time-resolved PL measurements, because most of the carrier concentrations that cause the blue shift in the PL peak position are in the range of carrier concentrations that exist during the PL decay tails.

The calculated ground state transition energy is compared with the blue shift in the PL peak position as a function of the excess carrier concentration in Fig. 3(a). The calculated ground state transition energy increases by $54 \mathrm{meV}$, which is in reasonable agreement with the $40 \mathrm{meV}$ blue shift observed in the PL peak position. The larger blue shift of the measured PL peak position at low carrier concentrations could be caused by filling of the tail states below the band edges, which is not considered in the calculations. The smaller blue shift of the measured PL peak position at large carrier concentrations could be attributed to a decrease of carrier lifetime due to the increase of wave function overlap in the type-II superlattice, the direct band-to-band transition in CdSe or a heating effect at the high excitation levels.

Fig. 3(b) shows the maximum PL peak intensity as a function of excess carrier concentration. For excess carrier concentrations of less than $3 \times 10^{17} \mathrm{~cm}^{-3}$, the SRH recombination dominates and, therefore, the PL signal increases superlinearly with the excess carrier concentration. As the excess carrier concentration increases, the PL internal quantum efficiency increases until the peak intensity is directly proportional to excess carrier concentration as shown by the dashed line, because the competing SRH process is saturated. The PL intensity increases sublinearly when the excess carrier concentration becomes larger than $9 \times 10^{17} \mathrm{~cm}^{-3}$. This behavior could be caused by spatially direct band-toband recombination in $\mathrm{CdSe}$, which is out of the detection range of the Ge detector, or by the heating effect at the high excitation levels. These results indicate that the carrier lifetime may start to decrease at the excess carrier concentration of $3 \times 10^{17} \mathrm{~cm}^{-3}$ because the radiative recombination becomes dominant. Note that the decrease of carrier lifetime happens at a slightly higher carrier concentration in the PL decay with an initial excess carrier concentration of $5 \times 10^{18} \mathrm{~cm}^{-3}$. This discrepancy could be due to errors in the estimation of carrier concentrations for these two measurements.

In summary, the optical properties of a type-II CdTe/ CdSe superlattice have been studied using time-resolved

TABLE I. Material parameters for zinc-blende CdTe and CdSe [ $a$ : lattice constant, $E_{g}$ : bandgap energy, VBO: valence band offset, $m_{\mathrm{e}}$ : electron effective

\begin{tabular}{|c|c|c|c|c|c|c|c|c|}
\hline & $a(\AA)$ & $E_{g}(\mathrm{eV})$ & VBO (eV) (Ref. 4) & $m_{\mathrm{e}}$ & $m_{\mathrm{hh}}^{\mathrm{t}}$ & $m_{\mathrm{hh}}^{\mathrm{z}}$ & $m_{\mathrm{lh}}^{\mathrm{t}}$ & $m_{\mathrm{lh}}^{\mathrm{z}}$ \\
\hline $\mathrm{CdSe}$ & 6.052 (Ref. 9) & 1.76 (Refs. 9 and 10) & 0.63 & 0.12 (Ref. 14) & 0.17 (Ref. 17) & 0.83 (Ref. 17) & 0.36 (Ref. 17) & 0.13 (Ref. 17) \\
\hline \multirow[t]{2}{*}{ CdTe } & $\begin{array}{l}6.482 \text { (Refs. } \\
9,11 \text {, and } 12 \text { ) }\end{array}$ & $\begin{array}{c}1.61 \text { (Refs. } \\
9,12 \text {, and } 13)\end{array}$ & & 0.088 (Ref. 15) & $\begin{array}{l}0.14 \text { (Refs. } \\
15 \text { and } 16)\end{array}$ & $\begin{array}{l}0.53 \text { (Refs. } \\
15 \text { and } 16)\end{array}$ & $\begin{array}{l}0.28 \text { (Refs. } \\
15 \text { and } 16)\end{array}$ & $\begin{array}{l}0.11 \text { (Refs. } \\
15 \text { and } 16)\end{array}$ \\
\hline & $a_{\mathrm{c}}(\mathrm{eV})($ Ref. 18) & $a_{\mathrm{v}}(\mathrm{eV})($ Ref. 18) & $b(\mathrm{eV})($ Ref. 19) & $\varepsilon$ & \multicolumn{2}{|c|}{$\mathrm{C}_{11}\left(10^{11} \mathrm{dyne} / \mathrm{cm}^{2}\right)$} & \multicolumn{2}{|c|}{$\mathrm{C}_{12}\left(10^{11}\right.$ dyne $\left./ \mathrm{cm}^{2}\right)$} \\
\hline $\mathrm{CdSe}$ & -3.77 & -1.81 & -0.8 & 9.6 (Ref. 19) & \multicolumn{2}{|c|}{8.8 (Ref. 11) } & \multicolumn{2}{|c|}{$5.3($ Ref. 11$)$} \\
\hline CdTe & -5.09 & -2.14 & -1 & 10.4 (Refs. 12 and 19) & \multicolumn{2}{|c|}{5.35 (Refs. 11, 12, and 20) } & \multicolumn{2}{|c|}{3.68 (Refs. 11,12 , and 20$)$} \\
\hline
\end{tabular}
mass, $m_{h h}^{z}\left(m_{l h}^{z}\right)$ and $m_{h h}^{t}\left(m_{l h}^{t}\right)$ : heavy-hole (light-hole) effective mass in the growth direction and in plane, $a_{\mathrm{c}}, a_{\mathrm{v}}$, and $b$ : deformation potentials, $\varepsilon:$ dielectric constant, and $C_{11}$ and $C_{12}$ : elastic stiffness constants]. 

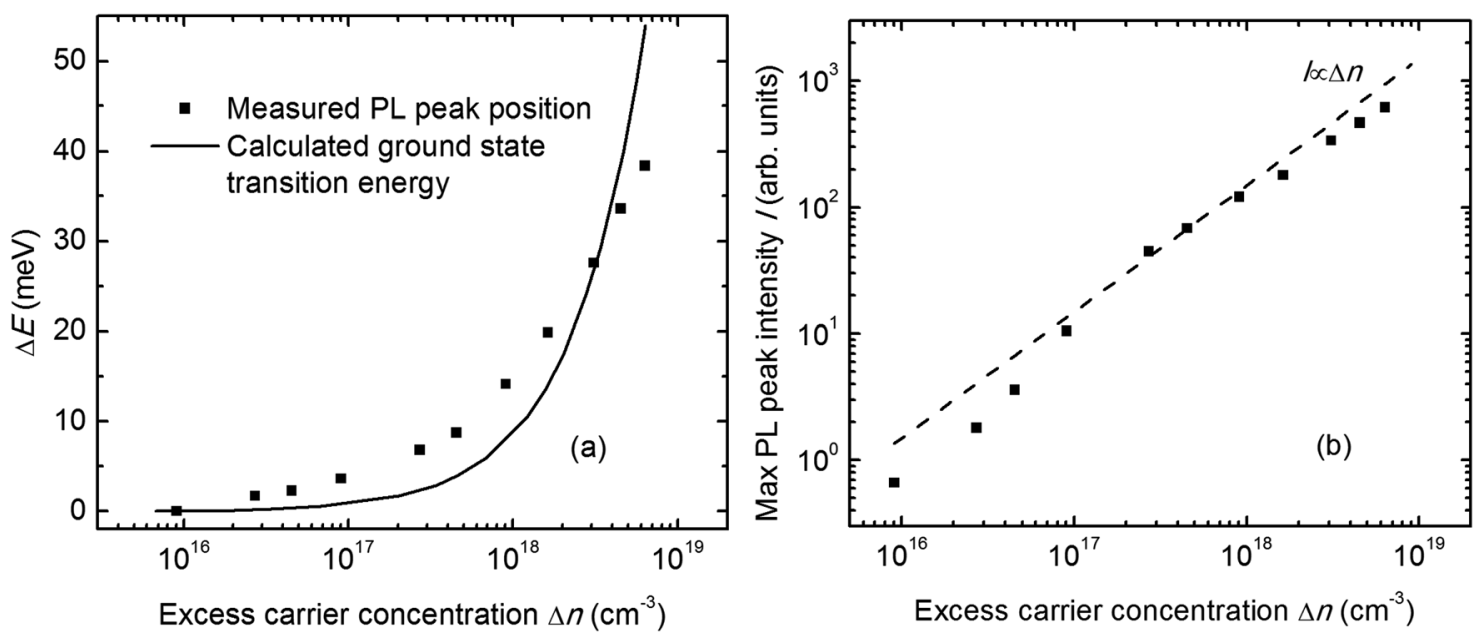

FIG. 3. (a) The blue shift $\Delta E$ in the measured PL peak position and the calculated ground state transition energy and (b) the maximum PL peak intensity, both as a function of the excess carrier concentration. The dashed line in part (b) shows the slope expected when the PL intensity is directly proportional to the excess carrier concentration.

photoluminescence and steady-state photoluminescence. The photoluminescence decay lifetime due to Shockley-Read-Hall recombination is obtained as $188 \mathrm{~ns}$. As a result of the type-II band alignment, the photoluminescence peak position shifts to shorter wavelengths with an increase of excess carrier concentration. A comparison of the measured blue shift with the increase in the ground state transition energy obtained from self-consistent solutions of the Schrödinger and Poisson equations suggests that this blue shift is primarily due to band bending associated with the type II band offsets.

This work was partially supported by the Science Foundation Arizona, Contract No. SRG 0339-08, the Air Force Research Laboratory/Space Vehicles Directorate, Contract No. FA9453-08-2-0228, and by NSF Grant ECCS-1002072. The authors would like to acknowledge Dr. Vijay D'Costa for the ellipsometry measurements.

${ }^{1}$ Y.-H. Zhang, S.-N. Wu, D. Ding, S.-Q. Yu, and S. R. Johnson, in Proc. 33rd IEEE Photovoltaic Specialist Conference (2008), p. 20.

${ }^{2}$ S. Wang, D. Ding, X. Liu, X.-B. Zhang, D. J. Smith, J. K. Furdyna, and Y.-H. Zhang, J. Cryst. Growth 311, 2116 (2009).
${ }^{3}$ S.-N. Wu, D. Ding, S. R. Johnson, S.-Q. Yu, and Y.-H. Zhang, Prog. Photovolt. Res. Appl. 18, 328 (2010).

${ }^{4}$ J.-J. Li, X. Liu, S. Liu, S. Wang, D. J. Smith, D. Ding, S. R. Johnson, J. K. Furdyna, and Y.-H. Zhang, Appl. Phys. Lett. 100, 121908 (2012).

${ }^{5}$ B. C. Connelly, G. D. Metcalfe, H. Shen, and M. Wraback, Appl. Phys. Lett. 97, 251117 (2010).

${ }^{6}$ N. N. Ledentsov, J. Böhrer, M. Beer, F. Heinrichsdorff, M. Grundmann, and D. Bimberg, Phys. Rev. B 52, 14058 (1995).

${ }^{7}$ C. H. Wang, T. T. Chen, K. W. Tan, Y. F. Chen, C. T. Cheng, and P. T. Chou, J. Appl. Phys. 99, 123521 (2006).

${ }^{8}$ K. T. Kim, S. S. Lee, and S. L. Chuang, J. Appl. Phys. 69, 6617 (1991).

${ }^{9}$ S.-H. Wei, S. B. Zhang, and A. Zunger, J. Appl. Phys. 87, 1304 (2000).

${ }^{10}$ W. Shan, J. Song, H. Luo, and J. Furdyna, Phys. Rev. B 50, 8012 (1994).

${ }^{11}$ E. Deligoz, K. Colakoglu, and Y. Ciftci, Physica B 373, 124 (2006).

${ }^{12}$ A. J. Strauss, Rev. Phys. Appl. (Paris) 12, 167 (1977).

${ }^{13}$ J. Camassel and D. Auvergne, Phys. Rev. B 12, 3258 (1975).

${ }^{14}$ Y. D. Kim, M. V. Klein, S. F. Ren, Y. C. Chang, H. Luo, N. Samarth, and J. K. Furdyna, Phys. Rev. B 49, 7262 (1994).

${ }^{15}$ L. S. Dang, G. Neu, and R. Romestain, Solid State Commun. 44, 1187 (1982).

${ }^{16}$ P. Lawaetz, Phys. Rev. B 4, 3460 (1971).

${ }^{17}$ M. Willatzen, M. Cardona, and N. Christensen, Phys. Rev. B 51, 17992 (1995).

${ }^{18}$ S.-H. Wei and A. Zunger, Phys. Rev. B 60, 5404 (1999).

${ }^{19} \mathrm{~S}$. Adachi, Properties of Group-IV, III-V and II-VI Semiconductors (Wiley, Chichester, 2005).

${ }^{20}$ H. J. McSkimin and D. G. Thomas, J. Appl. Phys. 33, 56 (1962). 